6. Brody JM, Butrus SI, Laby DM, Ashraf MF, Rabinowitz AI, Parenti DM. Anterior segment findings in AIDS patients with cytomegalovirus retinitis. Graefes Arch Clin Exp Ophthalmol 1995;233:374-6.

7. Wilhelmus KR, Font RL, Lehmann RP, Cernoch PL. Cytomegalovirus keratitis in acquired immunodeficiency syndrome. Arch Ophthalmol 1996;114:869-72.

8. Brown HH, Glasgow BJ, Holland GN, Foos RY.

Cytomegalovirus infection of the conjunctiva in AIDS. Am J Ophthalmol 1988;106:102-4.

9. Espana-Gregori E, Vera-Sempere FJ, Cano-Parra J, RamosFernandez V, Navea-Tejerina A, Diaz-Llopis M.

Cytomegalovirus infection of the caruncle in the acquired immunodeficiency syndrome. Am J Ophthalmol 1994;117:406-7.

Anita I. Miedziak

Christopher J. Rapuano

Cornea Service

Wills Eye Hospital

Thomas Jefferson University

Philadelphia

Pennsylvania, USA

Stephen Goldman

Department of Ophthalmology

Pennsylvania Hospital

Philadelphia

Pennysylvania, USA

Christopher J. Rapuano, MD

Cornea Service

Wills Eye Hospital

900 Walnut Street

Philadelphia

PA 19107, USA

Tel: $+1(215) 9283180$

Fax: +1(215) 9283854

Sir,

\section{Pedunculated episcleral choristoma without ocular involvement}

A choristoma is a congenital tumour-like growth that contains displaced epithelial and other dermis-like elements not normally indigenous to the site in which they are found. ${ }^{1}$ Choristomas can be divided into four main histopathological groups: dermoids, lipodermoids, single-tissue choristomas and complex choristomas. Dermoids consist of collagenous connective tissue covered by epidermoid epithelium. Lipodermoids contain adipose tissue along with a dermis-like connective tissue. Single-tissue choristomas consist of dermis-like tissue or ectopic tissues of mesoectodermal origin (lacrimal and other glands, fat, nerve, brain, cartilage, bone and teeth). Complex or composite choristomas contain tissues of different origins. ${ }^{2}$

Herein we present a rare case of a pedunculated episcleral choristoma with a very unusual presentation.

\section{Case report}

A 1-day-old Caucasian white male infant was referred to the Department of Ophthalmology at the Huddersfield Royal Infirmary with a pedunculated pink-coloured

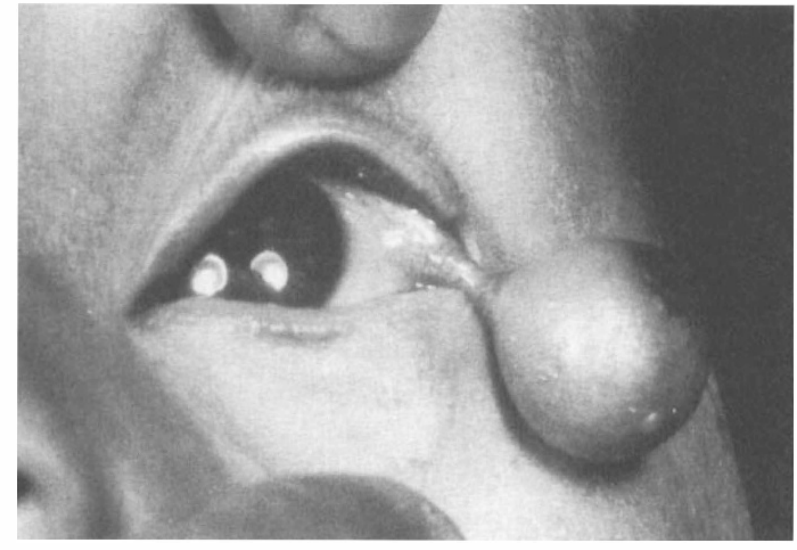

Fig. 1. A pedunculated episcleral mass protruding laterally through the left palpebral fissure.

mass arising from the temporal aspect of the left eye and hanging on the side of the face (Fig. 1). The child was full-term, with a birthweight of $4.12 \mathrm{~kg}$ and an Apgar score of 9 . The surface of the mass was covered with fine hair and a prominent scab could also be seen. The mass was excised under general anaesthesia.

Per-operatively, a haemostat was applied to the peduncle, followed by tying a ligature suture of $6 / 0$ catgut prior to excision of the mass. On excision, the peduncle seemed to retract into the conjunctival fornix. Ocular examination under anaesthesia revealed normal anterior and posterior segments with no evidence of any significant refractive error. An orthoptic assessment a few days later revealed apparently straight eyes with normal following. No skeletal abnormalities were found on radiography.

\section{Pathology}

On gross examination, the lesion consisted of a round pedunculated pink-coloured mass $9 \mathrm{~mm}$ in diameter. There was focal ulceration of the surface epithelium. Sagittal sectioning revealed fleshy yellow-coloured surfaces.

Histologically, the specimen was covered by a keratinised squamous epithelium. The tip of the nodule was ulcerated and covered by a fibrinopurulent material. The dermis contained numerous pilosebaceous follicles and occasional sweat glands. The centre of the specimen was occupied by a mature adipose tissue, intersected by fibrous septa. Many dilated vascular spaces were present within the dermis and the adipose tissue (Fig. 2, upper). At the base of the specimen there were well-defined aggregates of peripheral nerve tissue, intermixed with a mature cancellous bone, which was surrounded by osteoclasts (Fig. 2, lower).

\section{Comment}

Epibulbar choristomas can occur in isolation or with associated systemic malformations, which include Goldenhar's syndrome and mandibulofacial dysostosis (Treacher Collins syndrome and Franceschetti's 


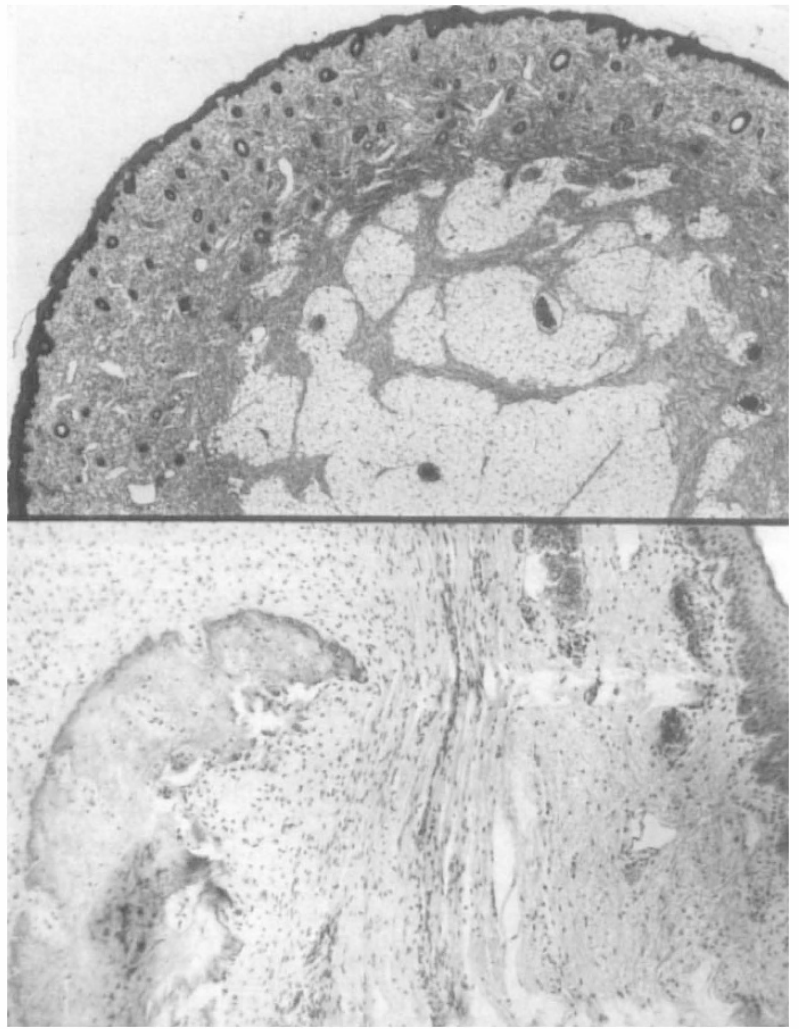

Fig. 2. Upper: Section of dermoid choristoma covered by skin with pilosebaceous apparatus and sweat glands. The centre of the specimen (left lower corner) shows mature adipose tissue, intersected by fibrous septae. HEE $\times 20$. Lower: A nodule of peripheral nerve tissue with central cancellous bone surrounded by osteoclasts. HEE, $\times 80$.

syndrome). ${ }^{1}$ Rumbaur ${ }^{3}$ in 1920 mentioned the possibility of epibulbar dermoids protruding through the palpebral fissure where they may become pedunculated. A similar case of a pedunculated epibulbar complex choristoma has been reported by Pe'er and Ilsar ${ }^{4}$ in association with naevus sebaceous and associated ocular anomalies, viz. choroidal colobomas and corneal vascularisation. Casey and Garner, ${ }^{5}$ in their case reports on epibulbar choristomas with microphthalmos, have suggested that intraocular involvement is quite a common association with these masses.

The possible pathogenesis of such an unusual pedunculated episcleral lesion is uncertain since it is poorly understood. The only hypothesis we could offer is that the evolution of this choristoma might have preceded the embryological fusion of the eyelids in the 12th week of gestation. The rationale for such an assumption is that the mass was lying outside the eye with a solitary communication with the temporal conjunctiva through the peduncle. This could suggest that once the palpebral fissure began to close, the lack of available space within the confines of the developing eyeball socket resulted in this mass protruding through the palpebral fissure. The outcome was a pedunculated mass, which continued to grow outside the eye. The presence of a notch in the lateral canthus of the left eye might suggest that the peduncle was lodged here, probably providing the necessary nourishment during development.
After reviewing most of the available literature we concluded that this case was unique in its clinical presentation, since it was an isolated anomaly with no ocular or systemic associations and it had an unual histological picture.

\section{References}

1. Albert MA, Jakobiec FA. Principles and practice of ophthalmology. Vol 1. Philadelphia: WB Saunders, 1994:277.

2. Mansour AM, Barber JC, Reinecke RD, Wang FM. Ocular choristomas. Surv Ophthalmol 1989;33:339-58.

3. Rumbaur. Klin Monatsbl Augenheilkd 1920;64:790.

4. Pe'er J, Ilsar M. Epibulbar complex choristoma associated with nevus sebaceus. Arch Ophthalmol 1995;1113:1301-4.

5. Casey RJ, Garner A. Epibulbar choristoma and microphthalmia: a report of two cases. Br J Ophthalmol 1991;75:247-50.

\section{J.L. Aggarwal}

H.S. Ahluwalia

H.H. Ali

Huddersfield Royal Infirmary

Huddersfield, UK

J.L. Aggarwal, FRCS, FRCOphth

Huddersfield Royal Infirmary

Lindley

Huddersfield HD3 3EA, UK

Sir,

\section{Conjunctival lymphangioma. A can of worms?}

We report a case of conjunctival lymphangioma that was unique in that its appearance was similar to Loa loa infestation. Excision and biopsy confirmed the nature of the lesion and there has been no recurrence to date. A review of lymphangiomas is presented.

\section{Case report}

A 42-year-old machinist presented to the Coventry Eye Unit with a right corneal foreign body that was removed under topical anaesthetic. An asymptomatic white curvilinear lesion was noted beneath the bulbar conjunctiva of the contralateral eye (Fig. 1). The patient

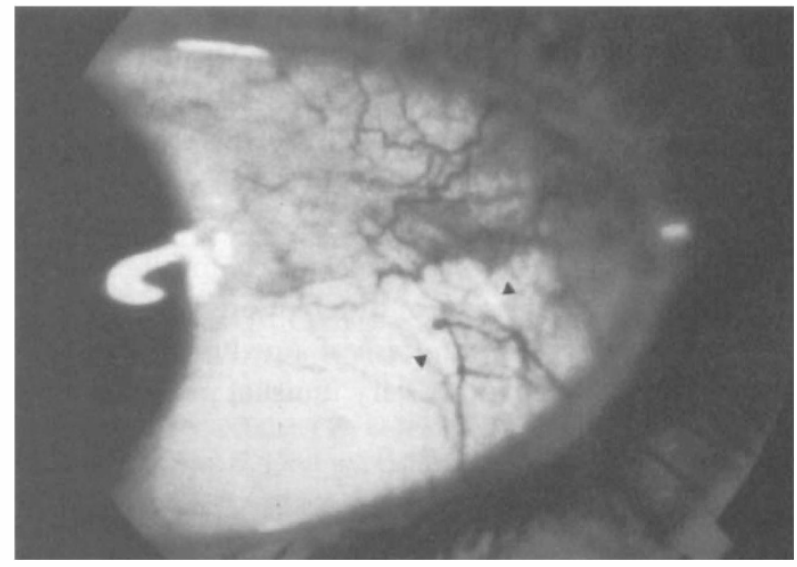

Fig. 1. Photograph showing the lesion beneath the temporal conjunctiva of the left eye. 